\title{
Neuronavigation-Assisted Aspiration and Electro-Acupuncture for Hypertensive Putaminal Hemorrhage: A Suitable Technique on Hemiplegia Rehabilitation
}

\author{
Yuhai ZHANG ${ }^{1}$, Rami AL-AREF², Haixia FU³ ${ }^{3}$ Yunxue YANG ${ }^{1}$, Yugong FENG4, Chao ZHAO ${ }^{1}$, Jun DONG ${ }^{1}$, \\ Guoqing SUN ${ }^{1}$ \\ ${ }^{1}$ Qingdao University, The Affiliated Hospital of Medical College, Department of Neurosurgery, Qingdao, China, \\ Current Work Unit: Ri Zhao Peoples Hospital, Department of Neurosurgery, Ri Zhao, Shan Dong Province, China \\ ${ }^{2}$ Wayne State University, School of Medicine, Department of Neurosurgery, Detroit, USA \\ ${ }^{3}$ Blood Purification Center, Affiliated Ri Zhao Peoples Hospital, Ji Ning Medical College, Ri Zhao, Shan Dong Province, China \\ ${ }^{4}$ Qingdao University, The Affiliated Hospital of Medical College, Department of Neurosurgery, Qingdao, China
}

\section{ABSTRACT}

AIM: To identify whether neuronavigation-assisted aspiration (NA) combined with electro-acupuncture (EA) provides better motor recovery in events of hypertensive putaminal hematoma (HPH) sized 30 to $50 \mathrm{ml}$. This study aims to examine whether neuronavigation-assisted aspiration and electro-acupuncture have additional value to cerebral hemorrhage motor rehabilitation.

MATERIAL and METHODS: 240 patients with HPH sized 30 to $50 \mathrm{ml}$ and admitted within 6 to 10 hours after stroke ictus were included in this study. Group 1 contained 60 patients who underwent neuronavigation-assisted aspiration and electro-acupuncture (NAEA), group 2 contained 60 patients who underwent neuronavigation-assisted aspiration (NA), group 3 contained 60 patients who underwent electro-acupuncture (EA), and group 4 contained 60 patients who received conservative therapy consisting solely of medications. All the patients received the same therapeutic plan on admission and functional exercises three days after stroke onset. Electro-acupuncture was performed on the third day of admission; motor recovery was examined on weeks zero and eight by blinded assessors. Outcome measures included Fugl-Meyer assessment, modified Ashworth Scale and Functional Independence Measure.

RESULTS: Group one showed significantly improved motor outcomes compared to group four ( $p<0.01)$. Group one also showed significant motor improvement when pre-and post- therapy functioning was examined $(p<0.01)$. Cerebral edema and ischemia were significantly decreased in group one compared to group 3 and $4(p<0.05)$. While not as effective as group one treatment, group two and group three patients had significant motor recovery after intervention when compared to group four ( $p<0.05)$. Muscular tension secondary to stroke was considerably improved between group one and group four, group two and group four, group three and group four respectively $(p<0.05)$. Activities of daily living $(A D L)$ improved a lot with EA together with NA.

CONCLUSION: Neuronavigation-assisted aspiration and electro-acupuncture of HPH at the early stage can provide improved motor recovery with fewer complications. Significant motor recovery can be achieved by neuronavigation-assisted aspiration with acupuncture. Based on our findings, we recommend early intervention with NA and EA in order to promote early rehabilitation of hemiplegia secondary to $\mathrm{HPH}$.

KEYWORDS: Neuronavigation-assisted aspiration, Hypertensive putaminal hematoma, Electro-acupuncture, Hemiplegia rehabilitation 


\section{INTRODUCTION}

$\mathrm{O}$ ne of the long-term clinical sequelae of hypertensive putaminal hematoma $(\mathrm{HPH})$ is severe hemiplegia. There is no clear consensus on operative management of $\mathrm{HPH}$ patients with multiple reports offering different operative techniques for treatment. Despite these multiple surgical techniques and current standard of therapy, a significant number of patients remain severely disabled after stroke.

Early studies suggested that surgical therapy did not provide improved motor recovery or mortality over conservative treatment in acute HPH (8). However, the presence of a hematoma causes massive damage to surrounding tissue by functioning as a large space-occupying lesion and creating an irreversible penumbra zone (27). It was found that the thrombin following intracerebral hemorrhage contributed to the formation of brain edema and secondary damage (18). These all serve to suggest that surgically evacuating an intracranial hemorrhage can potentially reduce neurological deficit and improve outcome. More specifically, minimally invasive surgery like stereotactic puncture aspiration under guided endoscopy and other similar procedures including those that are ultrasonic-, computed tomography (CT)-, and magnetic resonance imaging (MRI)-guided, are being advocated $(3,11,34)$. Conversely, decompression alone is insufficient as it results in increased incidence of recurrent postoperative hemorrhage. Neuronavigation-guided evacuation of $\mathrm{HPH}$, however, decreased injury to the cerebral structures while lessening edema and ischemia (21). Furthermore, electro-acupuncture has additional value in post-operative motor rehabilitation as it is purported to be associated with improved outcomes $(14,29)$. Previous studies showed that transcutaneous electrical stimulation to specific AccuPoints in specific limbs improved motor function after stroke through a mechanism of functional electrical stimulation $(14,24)$.

Our goal was to identify the role that neuronavigationassisted aspiration combined with electro-acupuncture plays as a therapeutic modality in patients after stroke. Generally, treatment is divided into two distinct stages with surgical intervention occurring in the acute stage and motorrehabilitation in the sub-acute stage. While it is clear that no single intervention can provide ideal results in the management of $\mathrm{HPH}$, we believe that neuronavigation-assisted aspiration and electro-acupuncture can significantly enhance motor recovery with this 10 -year prospective study.

\section{MATERIAL and METHODS}

\section{Patients}

A total of 240 consecutive patients with $\mathrm{HPH}$, who were selected over a span of ten years from January 2003 to December 2013 with the following criteria were included in this study. The diagnosis was determined by clinical presentation and CT scans. Patients with thalamic, lobar, or caudate hemorrhage were excluded. Any patients who had signs of pupillary changes (with one or both pupils indicating herniation) were excluded from the study. All patients were admitted within 6 hours of stroke. Other patient characteristics like age, gender, location of hemorrhage, volume of hematoma, and Glasgow Coma Scale (GCS) score were all recorded (Table I). The patients were divided into

Table I: Baseline Characteristics of the Stroke Patients

\begin{tabular}{|c|c|c|c|c|c|c|c|c|}
\hline \multirow[b]{2}{*}{ Characteristics } & \multicolumn{2}{|c|}{ Group $1(\mathrm{~N}=60)$} & \multicolumn{2}{|c|}{ Group $2(\mathrm{~N}=60)$} & \multicolumn{2}{|c|}{ Group $3(\mathrm{~N}=60)$} & \multicolumn{2}{|c|}{ Group $4(\mathrm{~N}=60)$} \\
\hline & $\mathbf{n}$ & $\%$ & $\mathbf{n}$ & $\%$ & $\mathbf{n}$ & $\%$ & $\mathbf{n}$ & $\%$ \\
\hline Age, (years) & \multicolumn{2}{|c|}{$57.92 \pm 9.6$} & \multicolumn{2}{|c|}{$56.72 \pm 8.3$} & \multicolumn{2}{|c|}{$59.65 \pm 10.7$} & \multicolumn{2}{|c|}{$56.35 \pm 9.2$} \\
\hline \multicolumn{9}{|l|}{ Sex } \\
\hline Male & 38 & $63.3 \%$ & 37 & $61.7 \%$ & 29 & $48.3 \%$ & 27 & $45 \%$ \\
\hline Female & 22 & $36.7 \%$ & 23 & $38.3 \%$ & 31 & $51.7 \%$ & 33 & $55 \%$ \\
\hline \multicolumn{9}{|l|}{ Bleeding side } \\
\hline Left & 21 & $35 \%$ & 24 & $40 \%$ & 26 & $43.3 \%$ & 32 & $53.3 \%$ \\
\hline Right & 39 & $65 \%$ & 36 & $60 \%$ & 34 & $56.7 \%$ & 28 & $46.7 \%$ \\
\hline \multicolumn{9}{|c|}{ Hematoma volume } \\
\hline$>40 \mathrm{ml}$ & 22 & $36.7 \%$ & 26 & $43.3 \%$ & 36 & $60 \%$ & 22 & $36.7 \%$ \\
\hline $30-40 \mathrm{ml}$ & 38 & $63.3 \%$ & 34 & $56.7 \%$ & 24 & $40 \%$ & 38 & $63.3 \%$ \\
\hline Mean & \multicolumn{2}{|c|}{45.3} & \multicolumn{2}{|c|}{44.6} & \multicolumn{2}{|c|}{47.2} & \multicolumn{2}{|c|}{43.9} \\
\hline \multicolumn{9}{|l|}{ GCS score } \\
\hline$<8$ & 19 & $31.7 \%$ & 22 & $36.7 \%$ & 28 & $46.7 \%$ & 24 & $40 \%$ \\
\hline$>8$ & 41 & $68.3 \%$ & 38 & $63.3 \%$ & 32 & $53.3 \%$ & 36 & $60 \%$ \\
\hline Mean & \multicolumn{2}{|c|}{9.7} & \multicolumn{2}{|c|}{10.3} & \multicolumn{2}{|c|}{9.6} & \multicolumn{2}{|c|}{10.2} \\
\hline
\end{tabular}

Group 1: neuronavigation and electro-acupuncture group; Group 2: neuronavigation group; Group 3: electro-acupuncture group; Group 4: conservative therapy group. 
four groups each containing 60 patients. Group 1 underwent neuronavigation-assisted aspiration and electro-acupuncture; group 2 underwent neuronavigation-assisted aspiration only; group 3 underwent electro-acupuncture only; and group 4 was managed with medication only. Patients were selected based on the presence of a hematoma located in the putamen with a volume of 30 to $50 \mathrm{ml}$ and a time interval of less than 10 hours between onset and operation. Cases in groups 1 and 3 received electro-acupuncture on day 3 of admission.

Electroacupuncture $(E A)$ is a useful therapy based on traditional acupuncture combined with modern electrotherapy that is currently being accepted as a treatment for ischemic stroke. Acupuncture needles were inserted into the veterinary accupoints, which are in charge of the affected functional area.

\section{Stratification, Randomization and Intervention}

First informed consent was obtained. For patients with a GCS score less than 8 the consent was obtained from the family (Code of Experimental Ethics: ChiCTR-TRC-11001671). Patients were assigned a random number by a computer that separated them into one of the four groups. Computergenerated random number tables and opaque envelopes were used to assign patients to group 1, group 2, group 3 or group 4. Patients allocated to group 1 received neuronavigation and electro-acupuncture, group 2 with neuronavigation, group 3 with electro-acupuncture and group 4 with conservative therapy, respectively.

Other than the principal investigator, attending neurosurgeon, and acupuncturist, the remainder of the team was blinded as to which groups the different patients were in. Specifically, therapists who performed the motor assessments were not involved in daily care and were blind to study design.

\section{Group 1}

Within the same time-period, group 1 patients were operated on using a minimally invasive method under the guidance of neuronavigation. The hematoma was aspirated through a frontal burr-hole with a drainage tube inserted into the cavity center of the hematoma.

Neuronavigation was employed to accurately establish the catheter surgical corridor (trajectory) across the frontal burrhole on the affected side. The target point and entry was set and a surgical plan was determined. Operative trajectory was parallel to the region with the longest diameter of the hematoma to the best of our ability.

The frontal entry point was located $3.2-4.6 \mathrm{~cm}$ from the middle line and $3.8-6.2 \mathrm{~cm}$ in front of the coronal suture. The target point was set in the latter $1 / 3$ of the hematoma; the drainage tube was inserted into the target point and slowly withdrawn to the center. The majority of the hematoma was aspirated using mild suction under assistance from the Vector Vision neuronavigation systems (Stealth Station Tria Plus, USA).

On day 3 of admission after initial aspiration, the patients in group 1 received scalp and body acupuncture. Patients with unstable vital signs or new-onset intracerebral hemorrhage were excluded until stabilized. Scalp and body acupuncture were performed at the same time with scalp acupuncture being used to localize motor reflex centers on the affected side. Body acupuncture was then performed on the paretic side. Main AccuPoints included (1) Ji quan, (2) Qu chi, (3) Shou san li, (4) He gu, (5) Wai guan, (6) Jian yu, (7) Jian liao (8) Bi nao, (9) Yang ling quan, (10) Zu san li, (11) Baihui, (12) Dazhui, (13) Cheng shan, and (14) San yin jiao $(5,35)$. These 14 points were selected on the basis of the Traditional Chinese Medicine theory (TCM).

These points are selected because they are closely related to the brain and spinal cord and these AccuPoints protect against hypoxic-ischemic brain damage (19) and facilitate the recovery of post-ischemic behavioral dysfunction (12).

Transcutaneous electrical stimulation to the AccuPoints on the affected limb was then administered. Therapeutic time was based on the standard acupuncture treatment of 2 sessions per day with 30 minutes per session (20). Electro-acupuncture therapy was continued for a period of 8 weeks. During times without therapy, the affected joints were maintained in a functional position.

\section{Group 2}

These patients only received neuronavigation-assisted aspiration following the protocol used on group 1 patients.

\section{Group 3}

These patients only received electro-acupuncture on day 3 of admission following the same protocol used in group 1.

\section{Group 4}

These patients received non-operative therapy that followed the standard medication protocol without electro-acupuncture or neuronavigation-assisted aspiration. The rehabilitation program was conducted from bedside and focused solely on functional exercises. These exercises were divided into whole body movement, motor skills, and motor coordination in order to avoid muscle atrophy and joint stiffness. During therapyfree times, the affected joints were maintained in a functional position.

\section{Ethical Considerations}

Participation in study was voluntary and patients were informed that they could withdraw at any point without need for explanation. Objective of the study along with potential complications were explained to the patients. Opportunity was provided for patients to ask questions. Patients gave their informed consent for participation along with assurance of confidentiality. Code of Experimental Ethics: ChiCTRTRC-11001671

\section{Measurements}

Fugl-Meyer Assessment of Physical Performance (FMA): FMA is a well-validated instrument assessing motor recovery after stroke, and has been widely used for both clinical and research purposes $(9,33)$. FMA score of the upper limb contained 66 points that we described along with its percentage of total in Table II. The total FMA score of the lower limb contained 34 points that we described along 
with its percentage of total in Table III. The motor domain has well-established reliability and validity as an indicator of motor impairment severity across different stroke recovery time points (28). FMA was assessed at 8 weeks by a blinded occupational therapist, who was unaware of which group the patient had been assigned to.

Modified Ashwort Scale (MAS): MAS was used to evaluate any changes in gastrocnemius muscular tension. Therapeutic effects of the various treatment modalities were evaluated 8 weeks after treatment using MAS and the limb motor function integration scale. A p-value of less than 0.05 was considered statistically significant.

Barthel index (BI): $\mathrm{BI}$ is the most widely used method for measuring the independence of daily activities in clinical settings. $\mathrm{BI}$ is evaluated on admission and 8 weeks after intervention to assess daily activity. A p-value of less than 0.05 was considered statistically significant.

\section{Statistical Methods}

Factorial design was used in this study. $x^{2}$ test, Mann-Whitney test, and two-factor analysis of variance were used to compare the demographic characteristics and other variables. The Mann-Whitney test was used to assess any difference in baseline FMA median scores and median score changes. Statistically significant or near-significant $(p<0.15)$ covariates, identified by Mann-Whitney test to be associated with FMA scores at 8 weeks, were further subjected to the generalized additive models (the non-parametric regression method) for multivariate analysis.

Analyses were performed using SPSS for Windows statistical software (version 15.0), and the S-Plus statistical program (version 8.0, MathSoft Inc). The level of significance was set at $5 \%$ in all comparisons, and all statistical testing was 2 -sided.

\section{RESULTS}

All $240 \mathrm{HPH}$ patients were enrolled within the first 10 hours of stroke onset. Baseline characteristics of the enrolled patients provided no significant variance among the four groups. During the trial, one patient from group 1 and two patients from group two withdrew secondary to coagulation disorders. Moreover, one patient from group 1 and three patients from group 3 passed away due to complications related to pulmonary, cardiac, and renal failure.

\section{Group 1}

Analysis after neuronavigation-assisted aspiration and electroacupuncture showed an increase of $18 \%$ or more with regards to the FMA score of the upper limb along with an increase of $41 \%$ to the FMA score of the lower limb. Moreover, a desirable $41.3 \%$ decrease of the MAS score gastrocnemius muscular tension was also observed.

\section{Group 2}

Analysis after neuronavigation-assisted aspiration showed a significantly diminished increase in the FMA score of both the upper and lower limb when compared to group 1. FMA score of the upper limb showed $8.65 \%$ increase while the FMA score of the lower limb was increased by $23.49 \%$. MAS score of gastrocnemius muscular tension decreased to $30.2 \%$, which was diminished when compared to group 1.

\section{Group 3}

Analysis after electro-acupuncture also showed a significantly

Table II: FMA Score of Upper Limb among the Four Groups $\left(\bar{X}_{ \pm s}\right)$

\begin{tabular}{llcccc}
\hline Group & $\mathbf{n}$ & Week 0 & (\%) & Week 8 & (\%) \\
\hline Group 1 & 60 & $9.51 \pm 7.66$ & 14.42 & $27.36 \pm 10.57$ & 41.52 \\
\hline Group 2 & 60 & $10.05 \pm 4.96$ & 15.89 & $20.79 \pm 6.38$ & 29.54 \\
\hline Group 3 & 60 & $9.75 \pm 6.64$ & 14.28 & $18.82 \pm 3.44$ & 28.53 \\
\hline Group 4 & 60 & $9.34 \pm 5.23$ & 14.56 & $12.27 \pm 8.21$ & 19.26 \\
\hline
\end{tabular}

$p<0.01$, comparison in Group 1 versus Group 4, pre and post treatment in Group 1. $p<0.05$, comparison in Group 1 versus Group 2, Group 1 versus Group 3, Group 2 versus Group 4, Group 3 versus Group 4, pre and post treatment in Group 2 and Group 3.

Table III: FMA Score of Lower Limb among the Four Groups $\left(\overline{\mathrm{X}}_{ \pm \mathrm{s}}\right)$

\begin{tabular}{llllll}
\hline Group & $\mathbf{n}$ & Week $\mathbf{0}$ & (\%) & Week 8 & (\%) \\
\hline Group 1 & 60 & $9.36 \pm 3.14$ & 26.72 & $24.37 \pm 9.16$ & 67.86 \\
\hline Group 2 & 60 & $9.88 \pm 1.42$ & 28.76 & $18.51 \pm 8.06$ & 52.25 \\
\hline Group 3 & 60 & $9.86 \pm 3.13$ & 28.71 & $17.82 \pm 6.65$ & 51.01 \\
\hline Group 4 & 60 & $9.23 \pm 4.36$ & 27.31 & $11.82 \pm 7.22$ & 33.03 \\
\hline
\end{tabular}

$p<0.01$, comparison in Group 1 versus Group 4, pre and post treatment in Group 1. $p<0.05$, comparison in Group 1 versus Group 2, Group 1 versus Group 3, Group 2 versus Group 4, Group 3 versus Group 4, pre and post treatment in Group 2 and Group 3. 
diminished increase in the FMA score of both the upper and lower limb when compared to group 1. FMA score of the upper limb showed $3.9 \%$ increase while the FMA score of the lower limb was increased by $12.2 \%$. MAS score of gastrocnemius muscular tension decreased $26.7 \%$, which was diminished when compared to group 1.

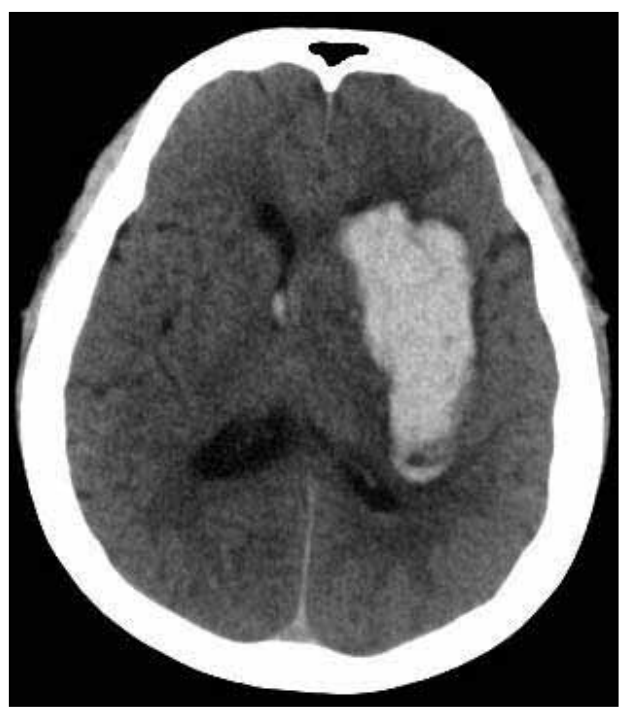

Figure 1:

Preoperative CT scan shows putaminal hemorrhage.

\section{Group 4}

Analysis after non-operative therapy showed a significantly diminished increase in the FMA score of both the upper and lower limb when compared to group 1. FMA score of the upper limb showed $0.7 \%$ increase while the FMA score of the lower limb was increased by $5.2 \%$. MAS score of gastrocnemius muscular tension decreased to $6.5 \%$ which was diminished when compared to group 1.

Based on these results, operative management with neuronavigation-assisted aspiration along with electro-acupuncture had the desired effect on motor rehabilitation after $\mathrm{HPH}$ (Figures 1-5). Statistical differences in the FMA score between pre and post intervention were significant in group 1 at a $\mathrm{p}<0.01$; these differences were also significant in group 2 and 3 at $p<0.05$. While the differences were considered significant in group 2 and 3 , they were diminished when compared to group 1. Score changes in MAS were also found to be significant between group 1 and group 4 at $p<0.01$ while also being significant being group 2 and group 3 at $p<0.05$ (Table IV).

Results from each of the different interventions indicate varying effects. Compared with group 2 and group 3, the combination of neuronavigation-assisted aspiration and electro-acupuncture showed significant advantage with regards to motor rehabilitation. That being said, both the interventions in group
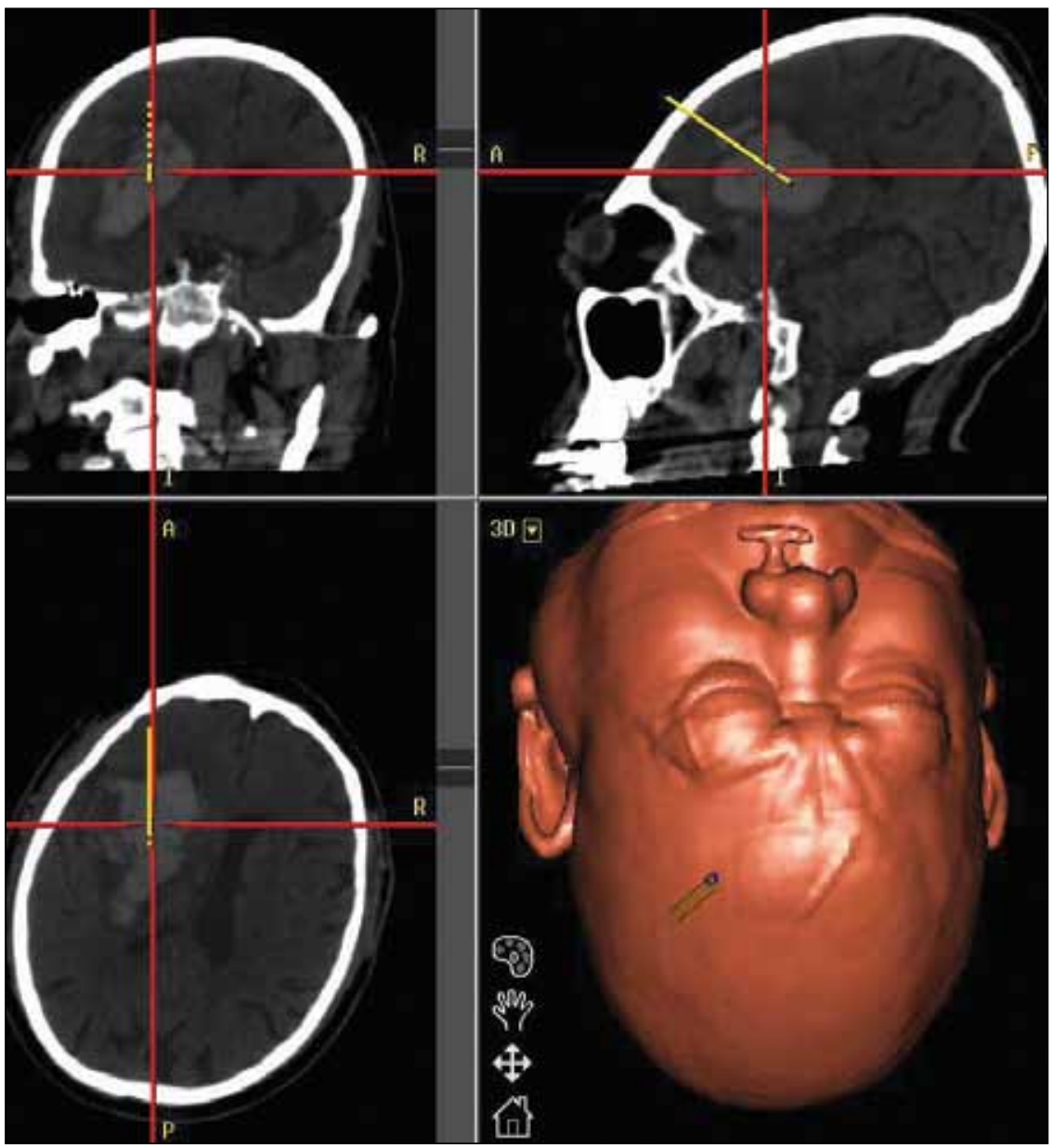

Figure 2: Intraoperative navigation image of the patient. 


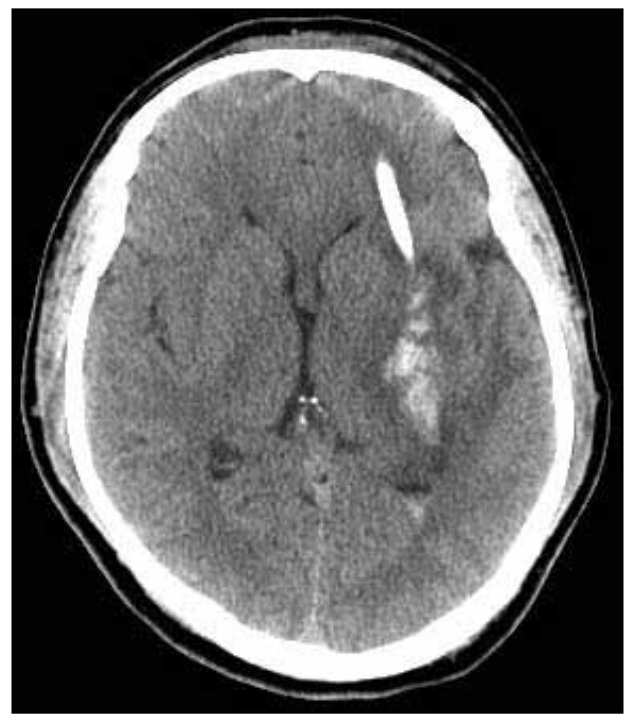

Figure 3:

CT scan

obtained at the postoperative $1^{\text {st }}$ hour.

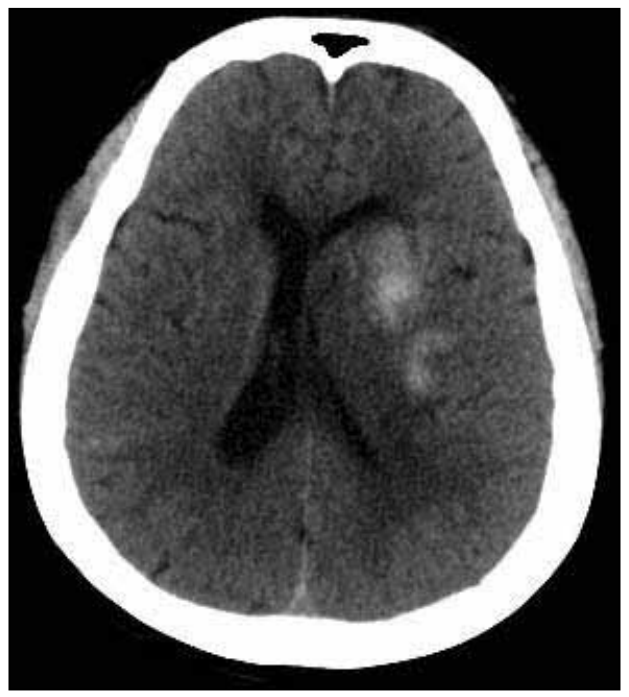

Figure 4: CT scan obtained at the $3^{\text {rd }}$ day after surgery.

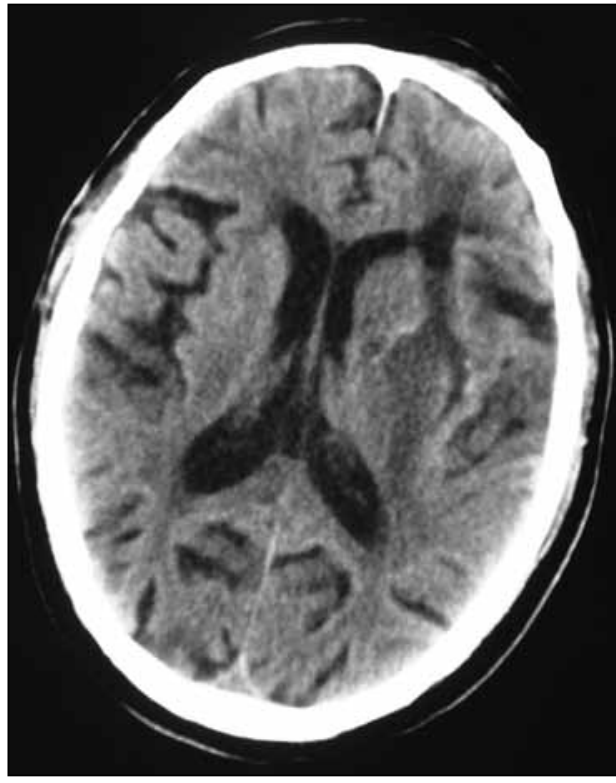

Figure 5: CT scan obtained at the $8^{\text {th }}$ week after surgery.
Table IV: MAS Score of Gastrocnemius Muscular Tension among the Four Groups $(\mathrm{X} \pm \mathrm{s})$

\begin{tabular}{lccc}
\hline Groups & $\mathbf{n}$ & Week 0 & Week 8 \\
\hline Group 1 & 60 & $3.86 \pm 1.11$ & $2.08 \pm 1.14$ \\
\hline Group 2 & 60 & $3.55 \pm 0.26$ & $2.39 \pm 0.38$ \\
\hline Group 3 & 60 & $3.71 \pm 0.56$ & $2.56 \pm 0.27$ \\
\hline Group 4 & 60 & $3.69 \pm 0.87$ & $3.45 \pm 1.21$ \\
\hline
\end{tabular}

$p<0.01$, comparison in Group 1 versus Group 4, pre and post treatment in Group 1. $P<0.05$ comparison in Group 1 versus Group 2, Group 1 versus Group 3, Group 2 versus Group 4, Group 3 versus Group 4, pre and post treatment in Group 2 and Group 3.

2 and group 3 did show distinct motor rehabilitation improvement over non-operative therapy in group 4 .

The most important measure however, was the percentage of subjects whose MAS score decreased by at least one grade after treatment. When comparing all the different groups, group 1 had the highest percentage of subjects whose spasticity improved by at least one grade $(p<0.01)$. To a lesser extent, a significant difference was also seen when comparing group 2 or group 3 with group $4(p<0.05)$ (Table IV).

The Barthel Index was increased significantly in the NA with EA group. This index also increased in the NA or EA group, but the changes were significantly less than in group 1 . Improved moving function and more flexible joints and ligaments were observed in comparison to the patients' condition prior to treatment.

\section{DISCUSSION}

Putaminal hemorrhage is the most common form of hypertensive intracerebral hematoma, presenting in around $70 \%$ to $80 \%$ of cases (22). Almost half of the deaths attributed to HPH are secondary to complications of the original hemorrhage including myocardial infarction, sudden death, extracranial hemorrhage and pneumonia (31). In most cases, no benefit with surgery has been reported for spontaneous supra-tentorial intracerebral hemorrhage $(4,15)$. However, when studies began to include data from the more modern post-CT era, a trend for improvement with surgery was found $(7,13)$. Moreover, minimally invasive methods of hematoma evacuation seem to show even more promising effects on patient outcomes and survival (32).

In a prior study, we showed that operation through a transsylvian-transinsular approach had promising effects. However, it was difficult to avoid secondary injury to the lateral fissure vessel resulting in brain edema with increased risk of infarction and worsening prognosis (36). Our previous study showed advantages over conventional craniotomy in that it can produce a clinically apparent decompressive effect. Despite this, it does not show any advantage over minimally invasive surgical evacuation especially with regards to secondary injury. The major disadvantage using the transsylvian- 
transinsular approach is that secondary brain injury is hard to avoid and may result in further brain damage if further surgery is required. Neuronavigation-assisted aspiration on the other hand, has major advantages in that it largely avoids secondary brain injury and allows for deep decompression of important neurostructures.

These present results suggest that motor rehabilitation benefited the most from immediate neural decompression using neuronavigation-assisted aspiration along with early transcutaneous electro-acupuncture. Moreover, minimally invasive surgery has significant advantages over conservative treatment in post-stroke motor recovery (Tables II, III). This is the first study to show that neuronavigation-assisted aspiration with electro-acupuncture as the best approach for the treatment of $\mathrm{HPH}$.

New strategies focusing on less invasive surgical techniques for clot evacuation are promising to decrease secondary neurologic injury. Recent studies have shown increased safety and feasibility of CT-guided thrombolysis and aspiration of intracranial hematomas $(23,25,30)$. Compared with older approaches, neuronavigation represents a more safe, effective, and minimally invasive operation.

Using this technique, we can freely choose the most efficacious entry site in order to remove the hematoma completely. Moreover, we can adjust our entry based on hematoma size and shape using imaging before the operation. Therefore, the best entry not only focuses on the center of the hematoma but also serves to avoid injuring important neurostructures like the internal capsule and surrounding vessels. The long-axis of the hematoma (geometrically determined using a cuboid shape) was always aligned to parallel the projected entry trajectory if the entry was frontal. However, if temporal entry was required, than the hematoma is geometrically determined as a round shape and aspiration is performed near the cortex. Using these techniques, one can essentially prevent damage to the posterior limb of the internal capsule.

Neuronavigation-assisted aspiration allows for more precise approaches to surgery, decreased surgical time, limited damage to surrounding structures, decreased morbidity, decreased infection rates, and most importantly shortened hospital stays $(2,16)$. In fact, when comparing surgical time between neuronavigation-assisted aspiration and a more traditional craniotomy, surgical time was decreased by approximately $60 \%$ from 2.75 to 1.05 hours. Since decompression in this case does not require a craniotomy, smaller incisions are adequate. Direct visualization of underlying structures during the operation allows the surgeon to make precise adjustments and avoid important structures (26). These unique advantages allow for decreased complications and improved clinical outcome in what is otherwise a devastating disease (6). When comparing differences in treatment modalities that were performed in this study, it is easy to see that significant improvement was seen between group 1 and group 3 . Both group 1 and group 3 had electro-acupuncture in the treatment plan; however group 1 also had neuronavigation-assisted aspiration which implies that it plays an important role in improving motor recovery. Coupled with decreased surgical time, risk of infections and other complications, this implies that neuronavigation-assisted aspiration is a more effective surgical option than the current standard of therapy.

During the sub-acute and chronic period post-operatively, effective therapy for motor rehabilitation is not always possible without adjuvant therapy. Most patients will inevitably suffer from some degree of muscular atrophy and stiffened joints further limiting rehabilitation. Prior reports suggest that hemiplegics can successfully restore motor function with targeted electrical stimulation of muscles in affected limbs (1).

Animal experiments proved that EA improved tissue and functional outcome following experimentally induced cerebral ischemia in mice. Endothelial nitric oxide synthase and muscarinic acetylcholine receptor-mediated perfusion augmentation might be related to these beneficial effects of EA (17). It is possible that enhancement of CBF might be the underlying mechanism of EA. A large number of animal studies have shown that EA could increase CBF in ischemic injury (10).

Statistically significant evidence that observed FMA scores on lower extremity motor function that provided credence to the use of electro-acupuncture as an adjunctive therapy in poststroke rehabilitation programs (1). Acupuncture helps achieve more flexible joints and allows for more mobility and inherently decreases risk of muscular atrophy and stiffening that are common in post-stroke motor rehabilitation.

Acupuncture is an alternative measure to enhance motor recovery and improve ADL in post-stroke patients. In other words, it functions as an effective technique to improve quality of life. Based on this and other studies, it is clear that transcutaneous electrical stimulation with task-related training improves motor function in a safe and effective manner while decreasing impairment and improving function in an individual with long-term, chronic stroke.

Based on the experimental data we gathered here, it is reasonable to think that functional motor recovery is significantly improved in patients with HPH after treatment with both neuronavigation-assisted aspiration and electroacupuncture. Specifically due to compression of the internal structure, the ability to actively visualize decompression of the internal capsule concurrent with early electro-acupuncture provides for improved motor recovery. Therefore, we strongly advocate decompression using neuronavigation-assisted aspiration and electro-acupuncture as major cornerstones of treatment plans in $\mathrm{HPH}$.

A true double-blinded acupuncture trial would be difficult to evaluate. In the present study, we used independent, blinded assessors for outcome measurement to avoid assessor bias. Therapists were also blinded as to which group the patient belonged to, to avoid therapist bias. Factors such as stroke status, nausea and vomiting, dysphagia, dysphasia, urinary incontinence, or a GCS score of 8 or less should prompt consideration of endotracheal intubation on admission. Moreover, the course of scalp acupuncture differs in patients who underwent neuronavigation-assisted aspiration and patients who did not, which also affects outcomes. In order 
to prevent these confounding factors, we accounted for these issues when performing our statistical analysis. While analyzing our data, we did not find any violations of our study design.

EA is helpful in the clinical therapy of stroke and accepted by some authors. However, more work is needed to determine the therapeutic window for EA to improve both tissue outcome and functional outcome. The precise mechanism through which this occurs is not fully understood and more evidence is needed for acute treatment with EA to be accepted clinically. Further studies will be needed to demonstrate the efficacy long-term acupuncture has in sustaining HPH motor recovery. Therefore, further investigation is needed to elucidate stroke subtypes and the therapeutic window within which NA and EA are efficacious.

Based on this information, we should take into consideration the limitations of this single-blinded control study when interpreting the results we presented. Moreover, this study was not concerned with other factors such as operation time, smoking history, cerebrovascular characteristics, compliance with therapy, diabetes, blood coagulation dysfunction, and limb thrombosis. In addition, several patients were lost to follow-up and this will inherently affect study results. In the paucity of rigorous trials concomitant with mixed results, we believe that our findings result in a limited conclusion. We suggest more rigorously designed and powered studies to be conducted.

\section{CONCLUSION}

We believe based on the data we presented that there can be significant functional motor recovery in patients who have experienced $\mathrm{HPH}$ if they are acutely treated with decompression using neuronavigation-assisted aspiration and then rehabilitated using electro-acupuncture. As an operative technique, neuronavigation-assisted aspiration is a safe, effective, and minimally invasive operation when compared to more standard therapies. EA is a useful method that provides cooperative action with NA in HPH patients and enhances their quality of life. This study provides the basis of a potential clinical model for treatment of HPH and improving functional motor recovery.

\section{ACKNOWLEDGEMENT}

Dr. Yonglin YANG made a greater contribution by collecting the pictures in this article.

\section{REFERENCES}

1. Alexander DN, Cen S, Sullivan KJ, Bhavnani G, Ma X, Azen SP: Effects of acupuncture treatment on poststroke motor recovery and physical function: A pilot study. Neurorehabil Neural Repair 18:259-267, 2004

2. Barnett $\mathrm{GH}$, Nathoo $\mathrm{N}$ : The modern brain tumor operating room: From standard essentials to current state-of-the-art. J Neurooncol 69:25-33, 2004
3. Barrett RJ, Hussain R, Coplin WM, Berry S, Keyl PM, Hanley DF, Johnson RR, Carhuapoma JR: Frameless stereotactic aspiration and thrombolysis of spontaneous intracerebral hemorrhage. Neurocrit Care 3:237-245, 2005

4. Batjer HH, Reisch JS, Allen BC, Plaizier LJ, Su CJ: Failure of surgery to improve outcome in hypertensive putaminal hemorrhage. A prospective randomized trial. Arch Neurol 47:1103-1106, 1990

5. Chang H, Kwon YD, Yoon SS: Use of acupuncture therapy as a supplement to conventional medical treatments for acute ischaemic stroke patients in an academic medical centre in Korea. Complement Ther Med 19:256-263, 2011

6. Chiou YH, Luh JJ, Chen SC, Chen YL, Lai JS, Kuo TS: Patient-driven loop control for hand function restoration in a non-invasive functional electrical stimulation system. Disabil Rehabil 30:1499-1505, 2008

7. Fernandes HM, Gregson B, Siddique S, Mendelow AD: Surgery in intracerebral hemorrhage. The uncertainty continues. Stroke 31:2511-2516, 2000

8. Ferro JM: Update on intracerebral haemorrhage. J Neurol 253:985-999, 2006

9. Fugl-Meyer AR: Post-stroke hemiplegia assessment of physical properties. Scand J Rehabil Med Suppl 7:85-93, 1980

10. Gao H, Guo J, Zhao P, Cheng J: The neuroprotective effects of electroacupuncture on focal cerebral ischemia in monkey. Acupunct Electrother Res 27:45-57, 2002

11. Grotta JC: Management of primary hypertensive hemorrhage of the brain. Curr Treat Options Neurol 6:435-442, 2004

12. Han $X$, Huang $X$, Wang $Y$, Chen $H$ : A study of astrocyte activation in the periinfarct region after cerebral ischemia with electroacupuncture. Brain Inj 24:773-779, 2010

13. Hayhurst C, Byrne P, Eldridge PR, Mallucci CL: Application of electromagnetic technology to neuronavigation: A revolution in image-guided neurosurgery. J Neurosurg 111:1179-1184, 2009

14. Hegyi G, Szigeti GP: Rehabilitation of stroke patients using Yamamoto New Scalp Acupuncture: A pilot study. J Altern Complement Med 18:971-977, 2012

15. Juvela S, Heiskanen O, Poranen A, Valtonen S, Kuurne T, Kaste $\mathrm{M}$, Troupp $\mathrm{H}$ : The treatment of spontaneous intracerebral hemorrhage. A prospective randomized trial of surgical and conservative treatment. J Neurosurg 70:755-758, 1989

16. Kaakaji W, Barnett GH, Bernhard D, Warbel A, Valaitis K, Stamp $S$ : Clinical and economic consequences of early discharge of patients following supratentorial stereotactic brain biopsy. J Neurosurg 94:892-898, 2001

17. Kim JH, Choi KH, Jang YJ, Bae SS, Shin BC, Choi BT, Shin HK: Electroacupuncture acutely improves cerebral blood flow and attenuates moderate ischemic injury via an endothelial mechanism in mice. PLoS One 8:e56736, 2013

18. Lee KR, Kawai N, Kim S, Sagher O, Hoff JT: Mechanisms of edema formation after intracerebral hemorrhage: Effects of thrombin on cerebral blood flow, blood-brain barrier permeability, and cell survival in a rat model. J Neurosurg 86:272-278, 1997 
19. Liu Y, Zou LP, Du JB, Wong V: Electro-acupuncture protects against hypoxic-ischemic brain-damaged immature rat via hydrogen sulfide as a possible mediator. Neurosci Lett 485:74-78, 2010

20. Li ZR: Experimental acupuncture science. Beijing, China.China Press of Traditional Chinese Medicine 37:124-137, 2003

21. Miller CM, Vespa PM, McArthur DL, Hirt D, Etchepare M: Frameless stereotactic aspiration and thrombolysis of deep intracerebral hemorrhage is associated with reduced levels of extracellular cerebral glutamate and unchanged lactate pyruvate ratios. Neurocrit Care 6:22-29, 2007

22. Mitchell P, Mitra D, Gregson BA, Mendelow AD: Prevention of intracerebral haemorrhage. Curr Drug Targets 8:832-838, 2007

23. Montes JM, Wong JH, Fayad PB, Awad IA: Stereotactic computed tomographic-guided aspiration and thrombolysis of intracerebral hematoma: Protocol and preliminary experience. Stroke 31:834-840, 2000

24. Ng SS, Hui-Chan CW: Transcutaneous electrical stimulation on acupoints combined with task-related training to improve motor function and walking performance in an individual 7 years poststroke: A case study. J Neurol Phys Ther 34:208213, 2010

25. Rohde V, Rohde I, Reinges MH, Mayfrank L, Gilsbach JM: Frameless stereotactically guided catheter placement and fibrinolytic therapy for spontaneous intracerebral hematomas: Technical aspects and initial clinical results. Minim Invasive Neurosurg 43:9-17, 2000

26. Shamir RR, Freiman M, Joskowicz L, Spektor S, Shoshan Y: Surface-based facial scan registration in neuronavigation procedures: A clinical study. J Neurosurg 111:1201-1206, 2009

27. Siddique MS, Fernandes HM, Wooldridge TD, Fenwick JD, Slomka P, Mendelow AD: Reversible ischemia around intracerebral hemorrhage: A single-photon emission computerized tomography study. J Neurosurg 96:736-741, 2002
28. Sullivan KJ, Tilson JK, Cen SY, Rose DK, Hershberg J, Correa A, Gallichio J, McLeod M, Moore C, Wu SS, Duncan PW: Fugl-Meyer assessment of sensorimotor function after stroke: Standardized training procedure for clinical practice and clinical trials. Stroke 42:427-432, 2011

29. Tan F, Wang X, Li HQ, Lu L, Li M, Li JH, Fang M, Meng D, Zheng GQ: A randomized controlled pilot study of the triple stimulation technique in the assessment of electroacupuncture for motor function recovery in patients with acute ischemic stroke. Evid Based Complement Alternat Med 2013:431986, 2013

30. Teernstra OP, Evers SM, Lodder J, Leffers P, Franke CL, Blaauw G: Stereotactic treatment of intracerebral hematoma by means of a plasminogen activator: A multicenter randomized controlled trial (SICHPA). Stroke 34:968-974, 2003

31. Vermeer SE, Algra A, Franke CL, Koudstaal PJ, Rinkel GJ: Long-term prognosis after recovery from primary intracerebral hemorrhage. Neurology 59:205-209, 2002

32. Wang GQ, Li SQ, Huang YH, Zhang WW, Ruan WW, Qin JZ, Li Y, Yin WM, Li YJ, Ren ZJ, Zhu JQ, Ding YY, Peng JQ, Li PJ: Can minimally invasive puncture and drainage for hypertensive spontaneous Basal Ganglia intracerebral hemorrhage improve patient outcome: A prospective non-randomized comparative study. Mil Med Res 1:10, 2014

33. Wood-Dauphinee SL, Williams JI, Shapiro SH: Examining outcome measures in a clinical study of stroke. Stroke 21:731739, 1990

34. Yamamoto T, Nakao Y, Mori K, Maeda M: Endoscopic hematoma evacuation for hypertensive cerebellar hemorrhage. Minim Invasive Neurosurg 49:173-178, 2006

35. Zhang XL, Qi R, Yan JT: Clinical research on post-stroke hemiplegia treated with the optimized rehabilitation program of integrated Chinese and western medicine. Zhongguo Zhen Jiu 33:1113-1117, 2013 (In Chinese)

36. Zhang Y, Ding W, Yang Y, Xu H, Xiong F, Liu C: Effects of transsylvian-transinsular approach to hypertensive putaminal hematoma operation and electroacupuncture on motor recovery. J Craniofac Surg 22:1626-1630, 2011 